ISBN978-93-86878-09-0

10th International Conference on Language, Humanities, Education and Social Sciences

(ICLHESS-18)

Bali (Indonesia) Jan. 12-13, 2018

\title{
A Study of the Broken Mirrors Phenomenon, and How it is Translated into High Fashion, Textiles, and Accessories
}

\author{
Dr. Sana Mahmoud Abbasi \\ Chair of the Fashion Design Department, Dar Al Hekma University, Jeddah, Saudi Arabia
}

\begin{abstract}
The Broken Mirrors Phenomenon is a belief that the broken mirror may result in seven years of bad luck, and is most likely one of the most well-known superstitions in the world. For many thousands of years people relied on highly polished metals as a reflective surface which held multiple purposes in daily life such as applying makeup, redirecting light, or even starting fires by focusing light into a fine point. In some cultures, any images caught in mirrors strangely, were believed that those images represented souls captured in another dimension. In the case of the Etruscan mirrors for example: If the mirror broke while someone was looking into it, the soul became trapped in many pieces, requiring either every single piece of the mirror to be ground into a fine dust or face seven years of bad luck. A soul is a direct reflection of who a person is. So it is the light within and an energy binding life to their existence. What's very interesting about mirrors and bad luck stories is how believers refer to the soul becoming trapped inside another dimension, almost alluding to a specific method for interacting or communicating with an alternative dimension. Broken mirrors is one of many superstitions around the world that is well known, however we find today high fashion Design houses such as the house of Dior making high end Dior bags with broken mirror's surface. This research paper will look into the relation between the Broken Mirror Phenomenon and how it is translated into Fashion, textiles, and accessories.
\end{abstract}

Keywords: Bad Luck, mirrors, evil eye, portal, superstation, Metaphysical Beliefs, negative entities, spirit, ghost, reflection, myths, Christian Dior

\section{Introduction}

Ever since human beings became aware of their own images, there has been fascinations and fears of reflective surfaces, such as the surfaces of the water, metals, and mirrors. Folklore and legends have many stories relating to magic and haunted mirrors (Sanofsky 2012). Most of them tend to send warning signs about the dangers of mirrors having supernatural qualities. However, mirrors were objects that it was believed that they can be utilized as portals to the spirit realm and even other dimensions. Some of the beliefs about mirrors that have been around for hundreds of years are that mirrors have the ability to suck out souls, and in order to avoid this from happening mirrors were removed from a room where an ill or dying person usually sleeps. Another belief was that people were warned never to look into a mirror at night or by candlelight because it was believed that one can see a ghosts, demons or signs of death. When a person died in a room the mirrors had to be covered or turned to face the wall. The failure to do so would result in the deceased person's soul being lost, or the possibility that they may turn into a vampire. Even while asleep, it was recommended to cover your mirror as you could be vulnerable to be attacked from negative spirits or demons during the night. It was also advised, to never place your bed in a place where it is reflected in a mirror. In order to prevent mirrors being used as a portal by supernatural entities, the mirror should be frequently moved to a different area of the room. Mirrors with a solid backing placed in the same position for a long period of time, are thought to be more likely to become spiritual portals. 


\section{Mystical Phenomena and Mirrors}

There are hundreds of documented cases of haunted mirrors and they tend to have similar characteristics. People have reported paranormal experiences when:

- An old mirror brought into the home from somewhere else

- People, when moving into a new home, have had problems with mirrors left behind by the previous occupants

- People have reported paranormal activity after using mirrors for divination, usually no protection was used by the participants and it is thought that they may have created a portal. It's suggested that when carrying out any kind of spiritual work with mirrors to use a cleansing and protective ritual. If you are doing spiritual work that doesn't involve a mirror, then these should either be covered or put away in a safe place

- Often people have reported having experiences with haunted mirrors in hotels, friends, and relative's home, visiting a place as a tourist and so on. These areas usually, but not always, have a reputation for being haunted.

The phenomena reported in connection with haunted mirrors are varied. The most common manifestation is the formation of images of people or entities other than the people occupying the room. However, it should be remembered that natural distortions and curious light effects can create a number of bizarre effects. Added to this is the brain's phenomenal ability to create meaningful shapes and faces out of random patterns. But despite this, many of the reported cases of haunted mirrors were witnessed by more than one person at different times of the day, in various lighting conditions. Therefore, these cases may possibly rule out natural causes. Added to this, other forms of paranormal manifestations developed in addition to that connected with the mirror involved.

\section{Negative Entities, Energy and Mirrors:}

The phenomena experienced by people are diverse. In most cases, but not all, the energy seems to be negative. However, some of the apparent malevolence might be due to a fear factor, suggestion or misinterpretation rather than a negative spirit.

Paranormal activity includes:

- Shadow People: They have frequently been witnessed in connection with haunted mirrors. Witnesses describe not only shadow people standing near to mirrors, but also within the mirror and entering or leaving them. Quite a few cases also reported other phenomena at the same time such as - cold spots, voices, noises and orbs. Odors - ranging from pleasant to vile - have also been described.

- Strange Mist: The mists are not only seen entering and leaving mirrors but sometimes form into more recognizable shapes - usually a human one. Other people have described these mists evolving into dense, dark masses that move around the room as well as within mirrors. The feelings reported by the witnesses are usually of a negative nature. Anger, hostility and evil are some of the feelings experienced. In addition, people have given accounts of other phenomena happening at the same time - cold spots, voices and spirit activity.

- Faces: This is probably by far the most common manifestation reported by people in association with haunted mirrors (Howell 2016). The faces are, most of the time, human - and sometimes known to the witnesses. But there are a number of accounts where people have reported other entities, and faces appearing. 


\section{Spirit Portals:}

Spirit portals are not a new concept and have been around for some time. At their most basic level, they are thought to be a form of entrance and exit point for spiritual energy and other dimensions. It has been claimed that portals have been captured on film, usually photographs.

\subsection{Characteristics of Some Portals:}

- A portal is thought to be a hole or window in the energy fields that surround the spiritual dimensions. Energy beings such as spirits can slip through these openings into the physical plane

- Negative spirits frequently come through portals. This is believed to happen because the astral layer closest to the physical plane is an area where negative energy and entities reside Therefore, when a window opens up, it's more likely for negative energy to pass through it first

- Most portals are believed to be a two way pathway for both entering and leaving the physical level.

- Very high levels of mystical activities are claimed to be found near portals

- Many believe that portals are found all over the world and there may even be certain hot spots

- Many believe that not only spiritual beings can use portals, but also aliens from other dimensions.

- It is thought that there are special, but very rare, portals that are completely positive. No lower astral or dimensional beings can enter these unless they are spiritually advanced.

- Some psychics and those who are developing their spirituality, claim that not only can they sense portals, but they can also close and open them up.

- It is thought by many that portals and tornados are the same thing. However, many psychics and investigators believe they are different. That a tornados is simply a pure band of energy, neither negative nor positive and is not a doorway. It can however, be used for meditation and other spiritual work.

- Not all psychics and paranormal investigators believe that portals exist

- Apart from mirrors, portals are thought to exist in areas of entry or exit within the physical realm. For example, places such as doorways, windows and even cupboards, wardrobes, are potentially portal areas

\section{Mirrors in Literature and Superstition:}

Mirrors have always possessed a certain spooky quality. Magic mirrors in literature since the ancient story of Narcissus, who fell in love with and pined for his own reflection in a pool of water, to present-day urban legends about summoning Bloody Mary by saying her name three times into a mirror. Snow White's queen had a magic mirror and Alice traveled through the looking glass to the other side. In ancient Chinese mythology, there's the story of the Mirror Kingdom, where creatures are bound by magic to sleep but will one day rise again to do battle with our world. Strange movements we see in mirrors out of the corners of our eyes are supposedly the first stirrings of this world as it wakes up. Mirrors also have a strong connection to the concept of the soul (Flinn 2009), which results in a wealth of superstition. For example, one of the reasons that breaking a mirror causes seven years of bad luck is that the soul, thought to regenerate every seven years, shatters with the broken mirror. Vampires, who have no souls, are thus invisible to mirrors. It's also dangerous for babies, whose souls are undeveloped, to look into mirrors before their first birthday or they will become stutterers. Spirits are often associated with mirrors. Mirrors are covered out of respect for the dead during the Jewish mourning ritual of sitting Shiva, but in many people in the U.S. also cover their mirrors when someone dies. According to superstition, a mirror can trap a dying soul. A woman who gives birth and looks in a mirror too soon afterward will also see ghostly faces peek out from behind her reflection. Rumor has it that if you go to a mirror on New Year's Eve with a candle in your hand and call out the name of a dead person in a loud voice, the power of the mirror will show you that person's face. 


\section{Three Myths about Mirrors in the Bedroom:}

There are three major myths about mirrors in the bedroom according to NIC Bambury, (July 15, 2017)

Myth \#1: Attracts a third party to the relationship: Mirrors across from the bed are believed to attract infidelity

Myth \#2: You startle yourself: Some Feng Shui enthusiasts believe that one can startle him or herself, if one awaken to his or her own reflection during the night.

Myth \#3: Issues with one's soul at night: This may sound far out to some of you so just bear with me. Some people believe that as we sleep, we transcend the physical plane. Having a mirror across from the bed might startle our soul as it leaves our body or may confuse our returning soul

\section{Reflection of the Soul}

Many ancient cultures thought reflections were not of your physical self, but of the very soul. Some cultures even believed that the mirror image at which one was staring revealed the shadow self-- the dark side of one's nature. This is why it was believed that evil creatures without a soul, such as vampires, did not have a reflection.

\section{Gateway to Other Worlds}

Many psychics and spiritualists have long held mirrors to be portals to other realms of existence. For example: Judaism is known for the tradition of covering the mirrors in a house during the mourning period after a loved one dies. It was believed that this tradition was developed so that the spirit wouldn't be trapped on earthly realms and could move to Heaven. During Victorian times, it was believed that by setting up mirrors to reflect each other, one could see the spirit world in the reflections. Urban legends have been convinced that spirits could be contacted through mirrors. Stories about what has happened to people who go into a dark room, look into a mirror and call a known and dangerous spirit several times. The most famous of these stories is Bloody Mary. As some found unexplained scratches on their bodies or experienced paranormal phenomenon for weeks after the stunt.

\subsection{Divination}

Some psychics and practitioners of magic use the reflective surface of a mirror by the light of a candle to induce visions. It is believed by these people that the mirror acts in the same way as a crystal ball, or a reflective surface is the ideal place for the mind to project the visions that come.

\subsection{Bad Luck}

Who doesn't shiver when accidentally breaking a mirror? It's been instilled into our consciousness that seven years of bad luck will follow. But this idea dates back to the belief that the mirror traps souls. When one breaks a mirror, it frees the angry, trapped souls from within who then have nowhere to go and nothing to do but to haunt the nearest person, and that is the person who broke the mirror.

\subsection{Good Luck}

Feng Shui, the Asian art of arranging a home for optimal flow of positive energy, has a lot to say about mirrors. Using mirrors to visually expand a room and bounce more light into it also expands fills it with beneficial energies. If there are any areas in the home where the energy gets trapped or doesn't flow properly, a carefully placed mirror can help redirect the energy to a more productive flow. In Feng Shui, mirrors are believed to bring calm and tranquility. They have the same effects as the elements of water, probably because their reflective surfaces are reminiscent of a still pond. Round mirrors are brought in to attract abundance and wealth to the home. 


\section{Fashion, Textiles, and Accessories created from Broken Mirrors:}

In the fall of 2017, the prime Lady Dior bag, recognized by its intricate leather stitching and distinctive metal charms, got an exhilarating makeover. From Swiss-Guinean artist Namsa Leuba's outlook on how African cultures are perceived in the West to John Giorno's thought-provoking poetry, each artist brings their distinct perspective to the maison's iconic accessory. The most impactful designs from the limited-edition series were chosen and are available at Dior boutiques nationwide starting December, 1, 2017

Lee Bul: Using an industrial interpretation of couture techniques, Lee Bul affixes dozens of tiny Plexiglas mirrors to the Lady Dior, simulating a single piece of shattered glass. The bag alludes to the artist's 2012 installation, "Cella," for the Miss Dior exhibition in Paris. The Dior Lady Art by Lee Bul was priced for $\$ 9700$ (Fig.1).

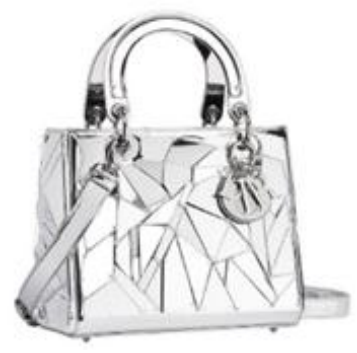

Fig. 1: Lee Bul Design for Dior House

Other fashion designers have created clothing using the broken mirrors such as Gucci fashion house that presented dresses with high-brilliance finishes that played with the light. A shower of metallic sequins, palettes and mosaics of tiny mirrors created a futuristic look for the trend of 2016.

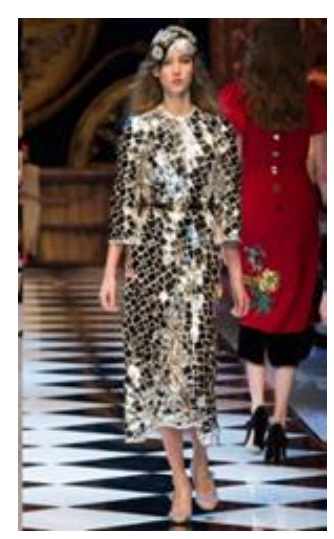

Fig. 2: Dress from Gucci Fall Collection 2016

Many jewelry and accessories designers also created amazing pieces using broken mirrors. For example: The theme of the "La Stanza dello Scirocco", designed by Mario Trimarchi, the successful series consisting of rectangular metal 'cards', has been expanded with the new Necklace, "Fiato sul collo" personal ornamentation, creating a typology of jewels. (Fig.3). Trimarchi was Director of Advanced Design at the Domus Academy and from 1989 to 2000 was part of the Olivetti Design Studio, where he worked with Michele De Lucchi. In 1999 he founded his own Corporate Identity Care studio, FRAGILE. With FRAGILE he designs systems of identity, coordinated image and visual alphabets through which diverse individualities can be expressed. 


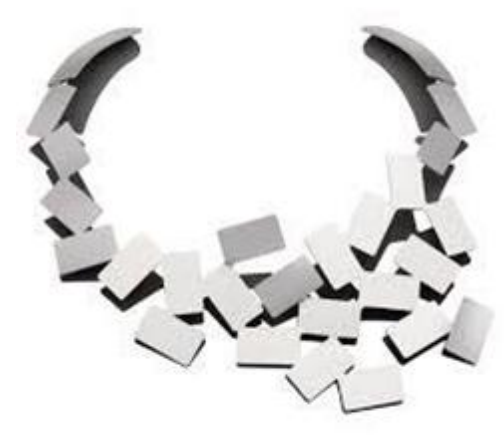

Fig.3: Necklace designed by: Mario Trimarchi for "La Stanza dello Scirocco",

\section{Conclusions:}

There are many myths and urban legends surrounding a simple household mirror. For example, it's generally believed that when one accidentally breaks a mirror, it would grant him or her seven years of bad luck. It is also believed that the origin for this myth is derived from the fact that, a mirror not only reflects one's physical appearance in its glass, but it would manifest a reflection of one's soul. If one accidentally breaks a mirror, that means that it also breaks one's soul, in which may expose this person to all the misfortune, because that person wouldn't have a soul that would protect him or her from the confusion. It's also believed that people may inhabit the mirror and study one's every movement. These people are jealous of one's life, and they would do anything in their power to switch places with the living person. There is also the famous Bloody Mary, the supposed ghost of a deceased witch was believed to appear in one's bathroom mirror. A person would go into his or her bathroom and proceed to close the door and turn off the bathroom lights. Then they would look into the mirror and say "Bloody Mary" three or thirteen times, then the ghost of Bloody Mary is supposed to appear in one's mirror. The soul would either kill the person on the spot, or she may pull the person into the mirror with her, and one will be trapped in there for eternity. As with any paranormal phenomena it's difficult to know which cases are supernatural or natural phenomena creating strange possessions. However, one thing is certain, mirrors will continue to play an important part in psychic phenomena.

With all these superstitions and negative energies and believes about the broken mirrors that it will bring seven years of bad luck, we find that high end Fashion Designers, and fashion houses create fashion products using broken mirrors, For example: The house of Dior has created the Dior bag with broken mirrors surface, designed by the artist Lee Bul, and Gucci House also created a complete collection of dresses, and accessories using broken mirrors. The jewelry Mario Trimarchi has designed a modern necklace based on the broken mirrors methodology. Fashion, textile, and jewelry designers, definitely are ignoring the superstitious, and the seven years of bad luck the broken mirrors may bring, and are looking at the positive side of this phenomenon, that will generate high income by creating something different!

\section{References}

[1] 1V. Edelstein, "Magneto-optical signature of broken mirror symmetry of two-dimensional conductors," JETP Letters, vol. 102 (11), 743-748, 2015

[2] The evil wiki, "Mirrors Superstitions", available: http://evil.wikia.com/wiki/Mirrors_(superstition)

[3] NIC Bambury, (July 15, 2017), Busting the 3 Myths about Mirrors in the Bedroom, available https://www.nicbambury.com/blog/busting-the-3-myths-about-mirrors-in-the-bedroom

[4] Josh Sanofsky, (Aug 27, 2012), A Time for Reflection: The Meaning of Mirrors in Folklore and Superstition, available http://weekinweird.com/2012/08/27/time-reflection-mirrors-folklore-superstition/ 
[5] Gallagher Flinn "How Mirrors Work", $\quad$ (Aug 4509$)$ HowStuffWorks.com. <https://science.howstuffworks.com/innovation/everyday-innovations/mirror.htm> 8 December 2017https://fengshuinexus.com/blog/mirror-facing-bed-feng-shui/

[6] Helen Murphy Howell, (April 15, 2016), "Spirit and Ghost Reflections in Haunted Mirrors," available: https://exemplore.com/paranormal/Spirit-Reflections-Ghosts-and-Haunted-Mirrors

[7] "Dior Lady Art 2 Project: Limited Edition Lady Dior Bags by 10 Different Artists," (Oct 25 2017), available: http://www.bagaddictsanonymous.com/2017/10/dior-lady-art-2-project-limited-edition.html

[8] “Trends of F/W 2016/2017," (Oct 13, 2016), available: https://mkfashionstyle.wordpress.com/2016/10/13/trends-offw-20162017/

[9] Alessi," available: https://www.alessi.com/us_en/product/person/jew/fiato-sul-collo.html 